Journal of New Results in Science
$\mathrm{https} / /$ dergipark.org.tr/en/pub/jnrs
Research Article
Open Access

\title{
Some results on generalized Euler-type integrals related to the four parameters Mittag-Leffler function
}

\author{
Umar Muhammad Abubakar1 ${ }^{1}$
}

\author{
Keywords: \\ Factorial function, \\ Pochhammer symbol, \\ Mittag-Leffler function, \\ Beta function, \\ Euler-type integral
}

\begin{abstract}
Special functions such as hypergeometric, zeta, Bessel, Whittaker, Struve, Airy, Weber-Hermite and Mittag-Leffler functions are obtained as a solution to complex differential equations in engineering, science and technology. In this work, generalized Euler-type integrals involving four parameters Mittag-Leffler function are proposed. Some special cases of this type of generalized integrals that are corresponding to well-known results in the literature are also inferred.
\end{abstract}

Subject Classification (2020): 33B15, 33B90.

\section{Introduction}

Throughout this article, $\mathbb{N}$ and $\mathbb{C}$ represent the sets of natural and complex numbers, respectively. In 1729, Leonard Euler studied the classical gamma function by extending the factorial function from the domain of natural numbers, to the region in the right half of the complex plane given as follows [1-2]:

$$
\Gamma(\sigma)=\int_{0}^{1} t^{\sigma-1} \exp (-t) d t, \quad(\operatorname{Re}(\sigma)>0)
$$

A year later, He established classical beta function, $B(\sigma, \mathcal{E})$ for a pair of complex numbers $\sigma$ and $\mathcal{E}$ with positive real parts through the integrand which is given by [3-4].

$$
B(\sigma, \mathcal{E})=\int_{0}^{1} t^{\sigma-1}(1-t)^{\mathcal{E}-1} d t, \quad(\operatorname{Re}(\sigma)>0, \operatorname{Re}(\mathcal{E})>0)
$$

Carl Friedrich Gauss [5] generalized the geometric series in the following classical Gauss hypergeometric function:

\footnotetext{
1umabubakar347@gmail.com

1Department of Mathematics, Faculty of Computing and Mathematical Sciences, Kano University of Science and Technology, Wudil, P.M.B: 3244 Kano, Kano State-Nigeria

Article History: Received: 03 June 2021 - Accepted: 25 Dec 2021 — Published: 31 Dec 2021
} 


$$
F(\sigma, \mathcal{E} ; \text { ð; } z)=\sum_{n_{2}}^{\infty} \frac{(\sigma)_{n_{2}}(\mathcal{E})_{n_{2}}}{(ð)_{n_{2}}} \frac{z^{n_{2}}}{n_{2} !}=\sum_{n_{2}}^{\infty}(\sigma)_{n_{2}} \frac{B\left(\mathcal{E}+n_{2}, \text { ठ }-\varepsilon\right)}{B(\mathcal{E}, \text { ð }-\mathcal{E})} \frac{z^{n_{2}}}{n_{2} !}, \quad(|z|<1, \operatorname{Re}(ð)>\operatorname{Re}(\mathcal{E})>0)
$$

and it can reduced to the Kumar confluent hyper geometric function defined by Ernst Eduard Kumar in [6].

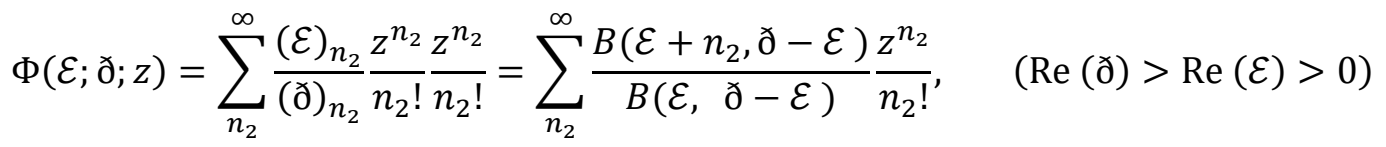

Here $(\sigma)_{n_{2}}$ represent classical pochhammer symbol defined as [7-8].

$$
(\sigma)_{n_{2}}=\left\{\begin{array}{cc}
\sigma(\sigma+1)(\sigma+2)(\sigma+3) \cdots\left(\sigma+n_{2}-1\right), & \left(n_{2} \geq 0, \sigma \neq 0\right) \\
1 & \left(n_{2}=0\right)
\end{array}\right.
$$

Other properties of Gauss hypergeometric and confluent hypergeometric such integral representation, transformation formulas, summation formulas and contiguity relations can be found in [9].

Chaudhry and Zubair [10-11] extended classical gamma function in (1.1) by using exponential kernel as follows [12-14]:

$$
\Gamma_{\Im}(\sigma)=\int_{0}^{1} t^{\sigma-1} \exp \left(-t-\frac{\mathfrak{J}}{t}\right) d t, \quad(\operatorname{Re}(\sigma)>0, \operatorname{Re}(\mathfrak{I})>0)
$$

Chaudhry et al., [15-17] introduced the following extension of beta function as an extension of classical beta function in (1.2):

$$
B_{\mathfrak{J}}(\sigma, \mathcal{E})=\int_{0}^{1} t^{\sigma-1}(1-t)^{\mathcal{E}-1} \exp \left(-\frac{\mathfrak{J}}{t(1-t)}\right) d t, \quad(\operatorname{Re}(\mathfrak{I})>0, \operatorname{Re}(\sigma)>0, \operatorname{Re}(\mathcal{E})>0)
$$

Chaudhry et al., [18] proposed the following extended of Gauss hypergeometic and confluent hyper geometric functions by using extended beta function in (1.3) and (1.4) as

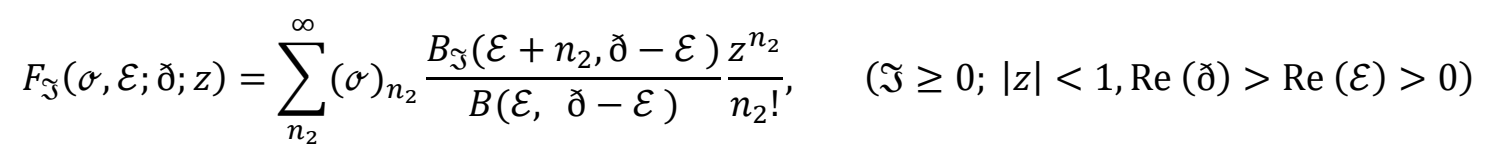

and

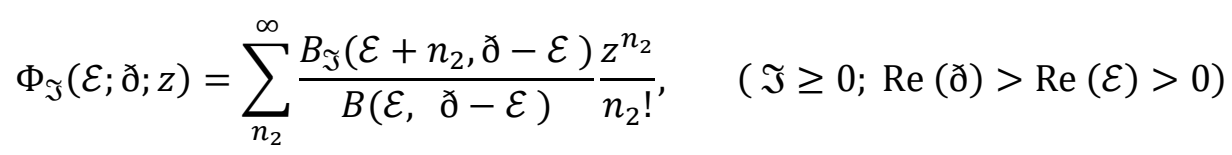

Lee et al., [19-20] presented and investigated the following extension of beta function as an extension of (1.5):

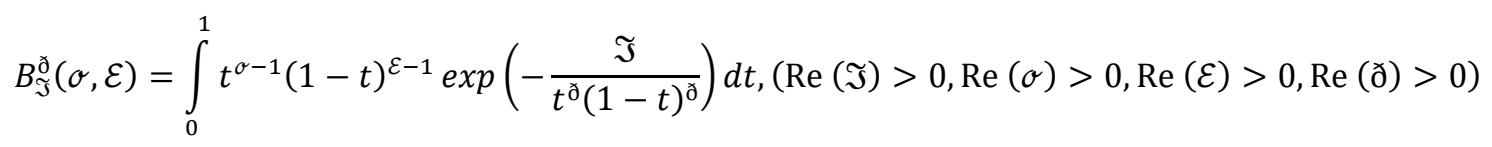


They also [19] extended Gauss and confluent hyper geometric functions in (1.6) and (1.7) as follows:



and

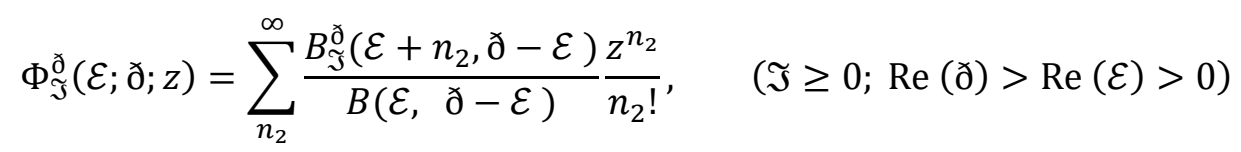

Luo et al., [21] presented the following extension of beta function as a generalization of beta function in (1.10):

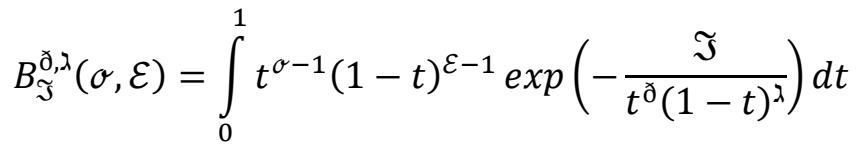

$$
\begin{aligned}
& (\operatorname{Re}(\Im)>0, \operatorname{Re}(\sigma)>0, \operatorname{Re}(\mathcal{I})>0, \operatorname{Re}(ð)>0, \operatorname{Re}(ג)>0)
\end{aligned}
$$

The classical Mittag-Leffler function was first studied by G.M. Mittag-Leffler [22-24] as an extension of exponential function as shown below:

$$
E_{\omega}(z)=\sum_{n_{2}=0}^{\infty} \frac{z^{n_{2}}}{\Gamma\left(\omega n_{2}+1\right)}, \quad(\omega, z \in \mathbb{C}, \operatorname{Re}(\omega)>0)
$$

Wiman [25-26] extended classical Mittag-Leffler function in (1.12) by introducing two parameters Mittag-Leffler function as follows:

$$
E_{\omega, \varpi}(z)=\sum_{n_{2}=0}^{\infty} \frac{z^{n_{2}}}{\Gamma\left(\omega n_{2}+\varpi\right)}, \quad(\omega, \varpi, z \in \mathbb{C} \operatorname{Re}(\omega)>0, \operatorname{Re}(\varpi)>0)
$$

Prabhakar [27] investigated three parameters Mittag-Leffler function as a generalization of (1.13) as follows:

$$
E_{\omega, \varpi}^{\eta}(z)=\sum_{n_{2}=0}^{\infty} \frac{(\eta)_{n_{2}}}{\Gamma\left(\omega n_{2}+\varpi\right)} \frac{z^{n_{2}}}{n_{2}}, \quad(\omega, \varpi, \eta, z \in \mathbb{C}, \operatorname{Re}(\omega)>0, \operatorname{Re}(\varpi)>0, \operatorname{Re}(\eta)>0)
$$

Shukla and Prajapati [28] investigated four parameters Mittag-Leffler function as an extension of (1.14) as follows:

$$
E_{\omega, \varpi}^{\eta, q}(z)=\sum_{n_{2}=0}^{\infty} \frac{(\eta)_{q n_{2}}}{\Gamma\left(\omega n_{2}+\varpi\right)} \frac{z^{n_{2}}}{n_{2}}, \quad(\omega, \varpi, \eta, z \in \mathbb{C}, \operatorname{Re}(\omega)>0, \operatorname{Re}(\varpi)>0, \operatorname{Re}(\eta)>0, q \in(0,1) \cup \mathbb{N})
$$

where $(\eta)_{q n_{2}}$ represents generalized pochhammer symbol defined by [29]

$$
(\eta)_{q n_{2}}=\frac{\Gamma\left(\eta+q n_{2}\right)}{\Gamma(\eta)}
$$

Related literature is also available in [30-34]. 


\section{Main Result}

The generalized Euler-type integrals involving the four parameters Mittag-Leffler function are presented in the following theorems and corollaries:

Theorem 2.1. If $\omega, \varpi, \eta, \sigma, \mathcal{E}, \mathfrak{J} \in \mathbb{C}, \operatorname{Re}(\omega)>0, \operatorname{Re}(\varpi)>0, \operatorname{Re}(\eta)>0, \operatorname{Re}(\sigma)>0, \operatorname{Re}(\varepsilon)>0$, $\operatorname{Re}(\mathfrak{I})>0$ and $q \in \mathbb{N}$, then

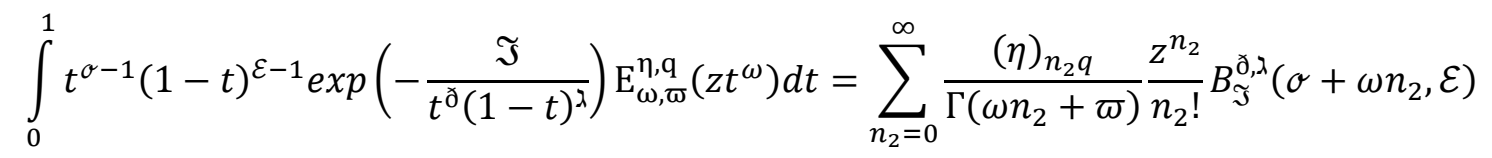

\section{Proof.}

Let represent left-hand side of (2.1) by $A_{1}$, we have

$$
A_{1}=\int_{0}^{1} t^{\sigma-1}(1-t)^{\mathcal{E}-1} \exp \left(-\frac{\widetilde{J}}{t^{ð}(1-t)^{\lambda}}\right) \mathrm{E}_{\omega, \varpi}^{\eta, \mathrm{q}}\left(z t^{\omega}\right) d t
$$

Applying (1.15), we obtain

$$
A_{1}=\int_{0}^{1} t^{\sigma-1}(1-t)^{\mathcal{E}-1} \exp \left(-\frac{\mathfrak{J}}{t^{ð}(1-t)^{\lambda}}\right)\left\{\sum_{n=0}^{\infty} \frac{(\eta)_{n_{2} q}}{\Gamma\left(\omega n_{2}+\varpi\right)} \frac{z^{n_{2}}}{n_{2} !} t^{\omega n_{2}}\right\} d t
$$

Interchanging the order of summation and integration, gives

$$
A_{1}=\sum_{n_{2}=0}^{\infty} \frac{(\eta)_{n_{2} q}}{\Gamma\left(\omega n_{2}+\varpi\right)} \frac{z^{n_{2}}}{n_{2} !} \int_{0}^{1} t^{\sigma+\omega n_{2}-1}(1-t)^{\mathcal{E}-1} \exp \left(-\frac{\mathfrak{I}}{t^{ð}(1-t)^{\lambda}}\right) d t
$$

Considering (1.11), we have

$$
A_{1}=\sum_{n_{2}=0}^{\infty} \frac{(\eta)_{n_{2} q}}{\Gamma\left(\omega n_{2}+\varpi\right)} \frac{z^{n_{2}}}{n_{2} !} B_{\Im}^{\text {ð,ג }}\left(\sigma+\omega n_{2}, \mathcal{E}\right)
$$

Lemma 2.2. The following result holds [35]:

$$
\begin{gathered}
\int_{a}^{b}(t-a)^{\sigma-1}(b-t)^{\mathcal{E}-1}(t v+h)^{g} d t=B(\sigma, \mathcal{E})(b-a)^{\sigma+\mathcal{E}-1}(a v+h)^{g}{ }_{2} F_{1}\left(\sigma,-g ; \sigma+\mathcal{E} ;-\frac{v(b-a)}{(a v+h)}\right) \\
\left(\operatorname{Re}(\sigma)>0, \operatorname{Re}(\mathcal{E})>0 ;\left|\arg \left((b v+h)(a v+h)^{-1}\right)\right|<\pi\right)
\end{gathered}
$$

Theorem 2.3. If $\omega, \varpi, \eta, \sigma, \mathcal{E}, \mathfrak{J} \in \mathbb{C}, \operatorname{Re}(\omega)>0, \operatorname{Re}(\varpi)>0, \operatorname{Re}(\eta)>0, \operatorname{Re}(\sigma)>0, \operatorname{Re}(\mathcal{E})>0$, $\operatorname{Re}(\mathfrak{J})>0,\left|\arg \left((b v+h)(a v+h)^{-1}\right)\right|<\pi$ and $q \in \mathbb{N}$, then 


$$
\begin{aligned}
& \int_{a}^{b}(t-a)^{\sigma-1}(b-t)^{\mathcal{E}-1}(t v+h)^{g} \exp \left(-\frac{\mathfrak{J}}{(t-a)^{ð}(b-t)^{\lambda}}\right) \mathrm{E}_{\omega, \mathrm{w}, \mathrm{q}}^{\eta}\left(z(b-t)^{\ell}\right) d t \\
& =(a v+h)^{g} \sum_{n_{1}}^{\infty} \sum_{n_{2}}^{\infty} \frac{(-\Im)^{n_{1}}}{n_{1}} \frac{(\eta)_{n_{2} q}}{\Gamma\left(\omega n_{2}+\varpi\right)} \frac{z^{n_{2}}}{n_{2} !} B\left(\sigma-ð n_{1}, \mathcal{E}-\lambda n_{1}+\kappa n_{2}\right) \\
& \times(b-a)^{\sigma+\mathcal{E}-ð n_{1}-\lambda n_{1}+\kappa n_{2}-1}{ }_{2} F_{1}\left(\sigma-ð n_{1},-g ; \sigma+\mathcal{E}-ð n-\lambda n_{1}+\kappa n_{21} ;-\frac{v(b-a)}{(a v+h)}\right)
\end{aligned}
$$

\section{Proof.}

Let donate left-hand side of (2.3) by $A_{2}$, we obtain

$$
A_{2}=\int_{a}^{b}(t-a)^{\sigma-1}(b-t)^{\mathcal{E}-1}(t v+h)^{g} \exp \left(-\frac{\widetilde{J}}{(t-a)^{ð}(b-t)^{\lambda}}\right) \mathrm{E}_{\omega, \varpi}^{\eta, \mathrm{q}}\left(z(b-t)^{\ell}\right) d t
$$

Using (1.11), we obtain

$$
A_{2}=\int_{a}^{b}(t-a)^{\sigma-1}(b-t)^{\mathcal{E}-1}(t v+h)^{g} \exp \left(-\frac{\mathfrak{J}}{t^{ð}(1-t)^{\lambda}}\right)\left\{\sum_{n_{2}=0}^{\infty} \frac{(\eta)_{n_{2} q}}{\Gamma\left(\omega n_{2}+\varpi\right)} \frac{\left(z(b-t)^{\ell}\right)^{n_{2}}}{n_{2} !}\right\} d t
$$

Considering the definition of exponential function

$$
\begin{aligned}
A_{2} & =\int_{a}^{b}(t-a)^{\sigma-1}(b-t)^{\mathcal{E}-1}(t v+h)^{g}\left\{\sum_{n_{1}=0}^{\infty} \frac{(-\Im)^{n_{1}}}{(t-a)^{n_{1} ð}(b-t)^{n_{1} \lambda} n_{1} !}\right\} \\
& \times\left\{\sum_{n_{2}=0}^{\infty} \frac{(\eta)_{n_{2} q}}{\Gamma\left(\omega n_{2}+\varpi\right)} \frac{\left(z(b-t)^{\ell}\right)^{n_{2}}}{n_{2} !}\right\} d t
\end{aligned}
$$

Interchanging the order of integration and summations, yields

$$
A_{2}=\sum_{n_{1}=0}^{\infty} \sum_{n_{2}=0}^{\infty} \frac{(-\mathfrak{J})^{n_{1}}}{n_{1} !} \frac{(\eta)_{n_{2} q}}{\Gamma\left(\omega n_{2}+\varpi\right)} \frac{z^{n_{2}}}{n_{2} !} \int_{a}^{b}(t-a)^{\sigma-n_{1} ð-1}(b-t)^{\mathcal{E}-n_{1} \lambda+n_{2} \ell-1}(t v+h)^{g} d t
$$

Applying (2.2) and simplifying, we get

$$
\begin{aligned}
A_{2} & =(a v+h)^{g} \sum_{n_{1}}^{\infty} \sum_{n_{2}}^{\infty} \frac{(-\Im)^{n_{1}}}{n_{1}} \frac{(\eta)_{n_{2} q}}{\Gamma\left(\omega n_{2}+\varpi\right)} \frac{z^{n_{2}}}{n_{2} !} B\left(\sigma-ð n_{1}, \mathcal{E}-\lambda n_{1}+\kappa n_{2}\right) \\
& \times(b-a)^{\sigma+\mathcal{E}-ð n_{1}-\lambda n_{1}+\kappa n_{2}-1}{ }_{2} F_{1}\left(\sigma-ð n_{1},-g ; \sigma+\mathcal{E}-ð n-\lambda n_{1}+\kappa n_{21} ;-\frac{v(b-a)}{(a v+h)}\right)
\end{aligned}
$$


Corollary 2.4. Substituting $\mathfrak{I}=0$, the following result can be obtained

$$
\begin{aligned}
& \int_{a}^{b}(t-a)^{\sigma-1}(b-t)^{\mathcal{E}-1}(t v+h)^{g} \exp \left(-\frac{\mathfrak{I}}{(t-a)^{\jmath}(b-t)^{\lambda}}\right) \mathrm{E}_{\omega, \mathrm{w}}^{\eta, \mathrm{q}}\left(z(b-t)^{\ell}\right) d t \\
& =(a v+h)^{g} \sum_{n_{2}=0}^{\infty} \frac{(\eta)_{n_{2} q}}{\Gamma\left(\omega n_{2}+\varpi\right)} \frac{z^{n_{2}}}{n_{2} !} B\left(\sigma, \mathcal{E}+\kappa n_{2}\right)(b-a)^{\sigma+\mathcal{E}+\kappa n_{2}-1} \\
& \times{ }_{2} F_{1}\left(\sigma,-g ; \sigma+\mathcal{E}+\kappa n_{2} ;-\frac{v(b-a)}{(a v+h)}\right)
\end{aligned}
$$

Corollary 2.5. Putting $a=0$ and $b=1$, the following formula can be obtained

$$
\begin{aligned}
& \int_{0}^{1}(t-a)^{\sigma-1}(b-t)^{\mathcal{E}-1}(t v+h)^{g} \exp \left(-\frac{\mathfrak{I}}{t^{ð}(1-t)^{\lambda}}\right) \mathrm{E}_{\omega, \varpi}^{\eta, \mathrm{q}}\left(z(1-t)^{\ell}\right) d t \\
& =h^{g} \sum_{n_{2}=0}^{\infty} \frac{(\eta)_{n_{2} q}}{\Gamma\left(\omega n_{2}+\varpi\right)} \frac{z^{n_{2}}}{n_{2} !} B\left(\sigma-ð n_{1}, \mathcal{E}-\lambda n_{1}+\kappa n_{2}\right)_{2} F_{1}\left(\sigma,-g ; \sigma+\mathcal{E}+\kappa n_{2} ;-\frac{v}{h}\right)
\end{aligned}
$$

Theorem 2.6. If $\omega, \varpi, \eta, \sigma, \mathcal{E}, \mathfrak{J} \in \mathbb{C}, \operatorname{Re}(\omega)>0, \operatorname{Re}(\varpi)>0, \operatorname{Re}(\eta)>0, \operatorname{Re}(\sigma)>0, \operatorname{Re}(\mathcal{E})>0$, $\operatorname{Re}(\mathfrak{I})>0 ; \alpha, \beta \geq 0$ and $q \in \mathbb{N}$, then

$$
\begin{aligned}
& \int_{0}^{1} t^{\sigma-1}(1-t)^{\mathcal{E}-\sigma-1}\left\{1-v t^{\alpha}(1-t)^{\beta}\right\}^{-\gamma} \exp \left(-\frac{\mathfrak{J}}{t^{ð}(1-t)^{\lambda}}\right) \mathrm{E}_{\omega, \varpi}^{\eta, \mathrm{q}}\left(z t^{\omega}\right) d t \\
& =\sum_{n_{1}}^{\infty} \sum_{n_{2}}^{\infty} \frac{v^{n_{1}}(\gamma)_{n_{1}}}{n_{1}} \frac{(\eta)_{n_{2} q}}{\Gamma\left(\omega n_{2}+\varpi\right)} \frac{z^{n_{2}}}{n_{2} !} B_{\Im}^{\text {ठ,ג }}\left(\sigma+\alpha n_{1}+\omega n_{2}, \mathcal{E}-\sigma+\beta n_{1}\right)
\end{aligned}
$$

\section{Proof.}

Let represent left-hand side of (2.4) by $A_{3}$, we have

$$
A_{3}=\int_{0}^{1} t^{\sigma-1}(1-t)^{\mathcal{E}-\sigma-1}\left\{1-v t^{\alpha}(1-t)^{\beta}\right\}^{-\gamma} \exp \left(-\frac{\mathfrak{I}}{t^{ð}(1-t)^{\lambda}}\right) \mathrm{E}_{\omega, \mathrm{w}}^{\eta, \mathrm{q}}\left(z t^{\omega}\right) d t
$$

Applying (1.15), we have

$$
A_{3}=\int_{0}^{1} t^{\sigma-1}(1-t)^{\mathcal{E}-\sigma-1}\left\{1-v t^{\alpha}(1-t)^{\beta}\right\}^{-\gamma} \exp \left(-\frac{\widetilde{J}}{t^{ð}(1-t)^{\lambda}}\right)\left\{\sum_{n_{2}=0}^{\infty} \frac{(\eta)_{n_{2}} q}{\Gamma\left(\omega n_{2}+\varpi\right)} \frac{z^{n_{2}}}{n_{2} !} t^{\omega n_{2}}\right\} d t
$$

Using binomial theorem, we get 


$$
\begin{aligned}
A_{3}= & \int_{0}^{1} t^{\sigma-1}(1-t)^{\mathcal{E}-\sigma-1}\left\{\sum_{n_{1}=0}^{\infty} \frac{v^{n_{1}} t^{\alpha n_{1}}(1-t)^{\beta n_{1}}}{n_{1} !}(\gamma)_{n_{1}}\right\} \exp \left(-\frac{\mathfrak{I}}{t^{ð}(1-t)^{\lambda}}\right) \\
& \times\left\{\sum_{n_{2}=0}^{\infty} \frac{(\eta)_{n_{2} q}}{\Gamma\left(\omega n_{2}+\varpi\right)} \frac{z^{n_{2}}}{n_{2} !} t^{\omega n_{2}}\right\} d t
\end{aligned}
$$

Interchanging the order of integration and summation, we have

$$
A_{3}=\sum_{n_{1}}^{\infty} \sum_{n_{2}}^{\infty} \frac{v^{n_{1}}(\gamma)_{n_{1}}}{n_{1}} \frac{(\eta)_{n_{2} q}}{\Gamma\left(\omega n_{2}+\varpi\right)} \frac{z^{n_{2}}}{n_{2} !} \int_{0}^{1} t^{\sigma+\alpha n_{1}+\omega n_{2}-1}(1-t)^{\mathcal{E}-\sigma+\beta n_{1}-1} \exp \left(-\frac{\mathfrak{J}}{t^{ð}(1-t)^{\lambda}}\right) d t
$$

Re-written this equation using (1.11), gives

$$
A_{3}=\sum_{n_{1}}^{\infty} \sum_{n_{2}}^{\infty} \frac{v^{n_{1}}(\gamma)_{n_{1}}}{n_{1}} \frac{(\eta)_{n_{2} q}}{\Gamma\left(\omega n_{2}+\varpi\right)} \frac{z^{n_{2}}}{n_{2} !} B_{\widetilde{J}}^{\delta, \lambda}\left(\sigma+\alpha n_{1}+\omega n_{2}, \mathcal{E}-\sigma+\beta n_{1}\right)
$$

Corollary 2.7. Setting $\gamma=0$ in (2.4), we have

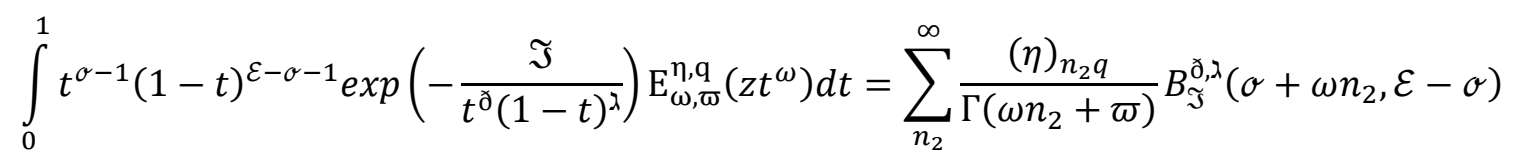

Corollary 2.8. Setting $\mathfrak{I}=\gamma=0$ in (2.4), we get

$$
\int_{0}^{1} t^{\sigma-1}(1-t)^{\mathcal{E}-\sigma-1} \mathrm{E}_{\omega, \varpi}^{\eta, \mathrm{q}}\left(z t^{\omega}\right) d t=\sum_{n_{2}}^{\infty} \frac{(\eta)_{n_{2} q}}{\Gamma\left(\omega n_{2}+\varpi\right)} B\left(\sigma+\omega n_{2}, \mathcal{E}-\sigma\right)
$$

\section{Conclusion}

In this work, we have proposed some generalized Euler type integrals involving four parameters MittagLeffler function of the form $E_{\omega, \varpi}^{\eta, q}(z)$ (refer to, [28]). In some special cases of this new generalized Euler type integrals includes:

Setting $ð=\lambda$ in (2.1), (2.3) and (2.4), we obtained the following Euler-type integrals that are in [36]:

$$
\begin{gathered}
\int_{0}^{1} t^{\sigma-1}(1-t)^{\mathcal{E}-1} \exp \left(-\frac{\mathfrak{J}}{t^{ð}(1-t)^{ð}}\right) \mathrm{E}_{\omega, \varpi}^{\eta, \mathrm{q}}\left(z t^{\omega}\right) d t=\sum_{n=0}^{\infty} \frac{(\eta)_{n q}}{\Gamma(\omega n+\varpi)} \frac{z^{n}}{n !} B_{\Im}^{ð}(\sigma+\omega n, \mathcal{E}) \\
\int_{a}^{b}(t-a)^{\sigma-1}(b-t)^{\mathcal{E}-1}(t v+h)^{g} \exp \left(-\frac{\mathfrak{J}}{(t-a)^{ð}(b-t)^{ð}}\right) \mathrm{E}_{\omega, \varpi}^{\eta, \mathrm{q}}\left(z(b-t)^{\ell}\right) d t \\
=(a v+h)^{g} \sum_{n_{1}}^{\infty} \sum_{n_{2}}^{\infty} \frac{(-\mathfrak{J})^{n_{1}}}{n_{1}} \frac{(\eta)_{n_{2} q}}{\Gamma\left(\omega n_{2}+\varpi\right)} \frac{z^{n_{2}}}{n_{2} !} B\left(\sigma-ð n_{1}, \mathcal{E}-ð n_{1}+\kappa n_{2}\right)
\end{gathered}
$$




$$
\times(b-a)^{\sigma+\mathcal{E}-2 ð n_{1}+\kappa n_{2}-1} F\left(\sigma-ð n_{1},-g ; \sigma+\mathcal{E}-2 ð n+\kappa n_{21} ;-\frac{v(b-a)}{(a v+h)}\right)
$$

and

$$
\begin{aligned}
& \int_{0}^{1} t^{\sigma-1}(1-t)^{\mathcal{E}-\sigma-1}\left\{1-v t^{\alpha}(1-t)^{\beta}\right\}^{-\gamma} \exp \left(-\frac{\mathfrak{J}}{t^{ð}(1-t)^{ð}}\right) \mathrm{E}_{\omega, \varpi}^{\eta, \mathrm{q}}\left(z t^{\omega}\right) d t \\
& =\sum_{n_{1}}^{\infty} \sum_{n_{2}}^{\infty} \frac{v^{n_{1}}(\gamma)_{n_{1}}}{n_{1}} \frac{(\eta)_{n_{2} q}}{\Gamma\left(\omega n_{2}+\varpi\right)} \frac{z^{n_{2}}}{n_{2} !} B_{\Im}^{ð}\left(\sigma+\alpha n_{1}+\omega n_{2}, \mathcal{E}-\sigma+\beta n_{1}\right)
\end{aligned}
$$

Finally, putting $ð=\lambda=1$ in (2.1), (2.3) and (2.4), we obtained the following Euler-type integrals that are in [37]:

$$
\begin{aligned}
& \int_{0}^{1} t^{\sigma-1}(1-t)^{\mathcal{E}-1} \exp \left(-\frac{\mathfrak{I}}{t(1-t)}\right) \mathrm{E}_{\omega, \varpi}^{\eta, \mathrm{q}}\left(z t^{\omega}\right) d t=\sum_{n=0}^{\infty} \frac{(\eta)_{n q}}{\Gamma(\omega n+\varpi)} \frac{z^{n}}{n !} B_{\mathfrak{J}}(\sigma+\omega n, \mathcal{E}) \\
& \int_{a}^{b}(t-a)^{\sigma-1}(b-t)^{\mathcal{E}-1}(t v+h)^{g} \exp \left(-\frac{\mathfrak{J}}{(t-a)(b-t)}\right) \mathrm{E}_{\omega, \varpi}^{\eta, \mathrm{q}}\left(z(b-t)^{\ell}\right) d t \\
& =(a v+h)^{g} \sum_{n_{1}}^{\infty} \sum_{n_{2}}^{\infty} \frac{(-\Im)^{n_{1}}}{n_{1}} \frac{(\eta)_{n_{2} q}}{\Gamma\left(\omega n_{2}+\varpi\right)} \frac{z^{n_{2}}}{n_{2} !} B\left(\sigma-n_{1}, \mathcal{E}-n_{1}+\kappa n_{2}\right) \\
& \quad \times(b-a)^{\sigma+\mathcal{E}-2 n_{1}+\kappa n_{2}-1} F\left(\sigma-n_{1},-g ; \sigma+\mathcal{E}-2 n+\kappa n_{21} ;-\frac{v(b-a)}{(a v+h)}\right)
\end{aligned}
$$

and

$$
\begin{aligned}
& \int_{0}^{1} t^{\sigma-1}(1-t)^{\mathcal{E}-\sigma-1}\left\{1-v t^{\alpha}(1-t)^{\beta}\right\}^{-\gamma} \exp \left(-\frac{\mathfrak{J}}{t(1-t)}\right) \mathrm{E}_{\omega, \omega}^{\eta, \mathrm{q}}\left(z t^{\omega}\right) d t \\
& =\sum_{n_{1}}^{\infty} \sum_{n_{2}}^{\infty} \frac{v^{n_{1}}(\gamma)_{n_{1}}}{n_{1}} \frac{(\eta)_{n_{2} q}}{\Gamma\left(\omega n_{2}+\varpi\right)} \frac{z^{n_{2}}}{n_{2} !} B_{\Im}\left(\sigma+\alpha n_{1}+\omega n_{2}, \mathcal{E}-\sigma+\beta n_{1}\right)
\end{aligned}
$$

Other form of special cases of this generalized Euler-type integral that are in the form of exponential, Classical Mittag-Leffler, Wiman and Prabhakar functions that are in [36-37] also follows.

\section{Conflicts of Interest}

The authors declare no conflict of interest.

\section{References}

[1] L.C. Andrews, Special functions of mathematics for engineers, SPIE Press, Bellingham, 1998.

[2] E.D. Rainville, Special functions, Macmillan, New York, 1960. 
[3] Y.L. Luke, The special functions and their approximations, Academic Press, New York, 1969.

[4] H.M. Srivastava, J. Choi, Zeta and q-zeta functions and associated series and integrals, Elsevier Amsterdam, 2012.

[5] I.N. Sneddon, Special functions of mathematical physics and chemistry, Edinburgh, London, New York,Interscience Publishers, New York, 1956.

[6] K.B. Oldham, J.C. Myland, J. Spaanier, An atlas of functions: with equator, the atlas function calculator, Springer, New York, 2009.

[7] U.M. Abubakar, S.R. Kabara, M.A. Lawan, F.A. Idris, A new extension of modified gamma and beta functions, Cankaya University Journal of Science and Engineering, 18 (1), (2021) 9-23.

[8] U.M. Abubakar, New generalized beta function associated with the Fox-Wright function, Journal of Fractional Calculus and Application, 12 (2), (2021) 204-227.

[9] R.K. Saxena, D.C. Gokhroo, Special functions, Jaipur Publishing House, Jaipur, 1987.

[10] M.A. Chaudhry, S.M. Zubair, Generalized incomplete gamma functions with applications, Journal of Computational and Applied Mathematics, 55, (1994)199-124.

[11] M.A. Chaudhry, S.M. Zubair, On decomposition of generalized incomplete gamma functions with applications to Fourier transform, Journal of Computational and Applied Mathematics, 59, (1995) 253-284.

[12] M.A. Chaudhry, S.M. Zubair, On extension of generalized incomplete gamma functions with applications, Journal of Australian Mathematical Society Series B, 37, (1996) 392-404.

[13] M.A. Chaudhry, Transformation of extended gamma function $\Gamma_{0,2}^{2,}[(B, X)]$ with applications to astrophysical thermonuclear functions, Astrophysics and Space Science, 262, (1999) 263-270.

[14] U.M. Abubakar, S.R. Kabara, $A$ note on a new extended gamma and beta functions and their properties, IOSR Journal of Mathematics, 15 (5), (2019) 1-6.

[15] M.A. Chaudhry, A. Qadir, M. Rafique, S.M. Zubair, Extension of Euler's beta function, Journal of Computational and Applied Mathematics, 78, (1997)19-32.

[16] M.A. Chaudhry, S.M. Zubair, On a class of incomplete gamma functions with application, Chapman \& Hall / CRC, New York, 2002.

[17] U.M. Abubakar, S.R. Kabara, Some results on the extension of the extended beta function, IOSR Journal of Mathematics, 15 (5), (2019) 7-12.

[18] M.A. Chaudhry, A. Qadir, H.M. Srivastava, R.B. Paris, Extended hyper geometric and confluent hyper geometric functions, Journal of Computational and Applied Mathematics, 159, (2004) 589602.

[19] D.M. Lee, A.K. Rathie, R.K. Parmar, Y.S. Kim, Generalization of extended beta function, hypergeometric and confluent hypergeometric functions, Homam Mathematical Journal, 33 (2), (2011)187-206.

[20] U.M. Abubakar, A study of extended beta and associated functions connected to Fox-Wright function, Journal of Fractional Calculus and Applications, 12(3), (2021), No:13, 1-23.

[21] M-J. Luo, G.V. Milovanovic, P. Agarwal, Some results on the extended beta and extended hypergeometric functions, Applied Mathematics and Computation, 248, (2014) 631-651. 
[22] G.M. Mittag-Leffler, Sur la nouvelle function $E_{\alpha}(x)$, Comptes Rendus de I'Academie des Sciences Paris Series II, 11 (137), (1903) 537-539.

[23] G.M. Mittag-Leffler, Soprala funzione $E_{\alpha}(x)$, Redicoti della Academia dei Lincei, V (13), (1904) 35.

[24] G.M. Mittag-Leffler, Sur larepresentation analytique d'une function monogone (inquieme note), Acta Mathematica, 29, (1905) 237-252.

[25] A. Wiman, Uber den fundamenta satz under theorie de function $E_{\alpha}(x)$, Acta Mathematica, 29, (1950) 191-201.

[26] A. Wiman, Uber die nullstellum de funktionen $E_{\alpha}(x)$, Acta Mathematica, 29, (1950) 217- 234.

[27] T.R. Prabhakar, $A$ singular integral equation with a generalized Mittag-Leffler function in the kernel, Yokohama Mathematical Journal, 19, (1971) 7-15.

[28] A.K. Shukla, J.C. Prajapati, On a generalization of Mittag-Leffler function and its properties, Journal of Mathematical Analysis and Applications, 336, (2007) 797-811.

[29] H.M. Srivastava, Z. Tomovski, Functional calculus with an integral operator containing a generalized Mittag-Leffler function in the kernel, Applied Mathematics and Computations, 211, (2009) 198-210.

[30] S. Khan, B. Agarwal, M.A. Pathan, F. Muhammad, Evaluation of Euler type integral, Applied Mathematics and Computation, 15, (2007), 31-49.

[31] S. Jabee, M. Shadab, R.B. Paris, Certain results on Euler-type integrals and their applications, Ramanujan Journal, 54, (2021), 245-260.

[32] M. Ali, W.A. Khan, I.A. Khan, Study on double integral operator involving generalized BesselMaitland function, Palestine Journal of Mathematics, 9 (2), (2020), 991-998.

[33] W.A. Khan, K.S. Nisar, M. Ahmad, Euler type integral operator involving K-Mittag-Leffler function, Boletim da Socidade Paranaense de Matematica, 38 (5), (2020), 165-174.

[34] M. Ghaysauddin, W.A. Khan, L.N. Mishra, On Hankle type integral transform associated with Whittaker and hypergeometric functions, Thai Journal of Mathematics, 19 (2), (2021), 521-527.

[35] A.P. Prudniko, Yu.A. Brychkov, O.I. Matichev, Integrals and series, Vol. 1, Gordan and Breach Science Publishers, New York, 1990.

[36] W.A. Khan, M. Ahmad, Some Euler type beta function involving extended Mittag-Leffler function, Palestine Journal of Mathematics, 9 (2), (2020) 969-976.

[37] S. Ahmed, M.A. Khan, Euler type integral involving generalized Mittag-Leffler function, Communication of Korean Mathematical Society, 29 (3), (2014) 479-487. 